\title{
A Tool for Optimizing the Use of a Large Design Space for Gesture Guiding Systems
}

\author{
William Delamare, Céline Coutrix and Laurence Nigay \\ CNRS, Joseph Fourier University UJF-Grenoble 1 \\ Grenoble Informatics Laboratory (LIG), UMR 5217, Grenoble, F-38041, France \\ \{William.Delamare, Celine.Coutrix, Laurence.Nigay\}@imag.fr
}

\begin{abstract}
We present a tool to help practitioners to characterize, compare and design gesture guiding systems. The tool can be used to find an example system meeting specific requirements or to start exploring an original research area based on unexplored design options. The motivation for the online tool is the large underlying design space including 35 design axes: the tool therefore helps explore and combine the various design options. Moreover the tool currently includes the description of 46 gesture guiding systems: the tool is thus also a repository of existing gesture guiding systems.
\end{abstract}

\section{Author Keywords}

Online Tool; Gesture Interaction; Guiding System; Design Space; Feedforward; Feedback.

\section{ACM Classification Keywords}

D.2.2. Software Engineering: Design Tools and Techniques - User interfaces.

\section{INTRODUCTION}

In order to optimize the use of our design space [5] for gesture guiding systems, we present an online tool available at http://iihm.imag.fr/delamare/guidance/.

A design space coherently structures a set of design issues for a particular class of interactive systems [1,3,4]. The resulting structure also provides a means for classifying existing systems. Three dimensions are proposed to characterize the effectiveness of a design space [2]: (1) its ability to describe a significant range of systems (descriptive power), (2) its ability to assess which design options are better (evaluative power) and (3), its ability to help design new systems (generative power). Any new design space aims at these three powers: descriptive, evaluative and generative. However, a large number of design options combined with a large number of existing

Permission to make digital or hard copies of part or all of this work for personal or classroom use is granted without fee provided that copies are not made or distributed for profit or commercial advantage and that copies bear this notice and the full citation on the first page. Copyrights for thirdparty components of this work must be honored. For all other uses, contact the Owner/Author.

Copyright is held by the owner/author(s).

EICS'15, June 23-26, 2015, Duisburg, Germany

ACM 978-1-4503-3646-8/15/06.

http://dx.doi.org/10.1145/2774225.2775440 systems can make it difficult for practitioners to efficiently use such design spaces. Indeed, classifying and describing a large number of systems along many design axes is a cumbersome task. In addition, a large, multidimensional design space, sometimes with multiple scales, makes it difficult to find unexplored areas.

Facing these issues, the proposed online tool interactively presents our large design space for gesture guiding systems: 35 axes characterizing 46 guiding systems (at the time of the submission). Gesture guiding systems are essential in order to reveal what commands are available and how to trigger them. The tool offers the following services that help designers and engineers to find the description of existing systems and to design new systems: (1) a description of existing systems one-by-one, (2) a high-level or detailed comparison of systems, (3) an interactive method to explore how often design options are used by existing systems and (4), a method to design a custom system and compare it to existing ones.

We first briefly recall the two main categories of the design space for which the tool has been built [5]. We then present the four services of the tool and illustrate how practitioners can use these services.

\section{UNDERLYING DESIGN SPACE}

The design space aims at characterizing the behavior of gesture guiding systems [5]. The design space is composed of 35 axes organized according to two categories: one dedicated to the feedback mechanism and one dedicated to the feedforward mechanism. These mechanisms provide information for guiding the user during gesture interaction. The feedback mechanism provides information regarding actions already performed by the user (i.e. the past). The feedforward mechanism provides information regarding actions available to the user (i.e. the future). Indeed, a gesture guiding system should reveal which commands are available and what the corresponding gestures are [6].

Each category (i.e. feedback and feedforward) contains four groups related to four questions about the guiding system:

- When (temporal characteristics), which contains design options about how the user triggers or exits the guide,

- What (content characteristics), which contains design options about the information content displayed by the guide, 
- How (medium characteristics), which contains design options about the way the content is presented to the user,

- Where (spatial characteristics), which contains design options about the physical location of the guide.

Overall, the design space includes 35 axes that identify 115 design options. 46 existing systems inspired from 24 information sources (e.g., research papers or commercial products) are described within the tool. Moreover a guiding system can include one or more steps. A step describes one phase of the guidance process and is described by feedback and feedforward aspects. The description of the 46 existing systems results overall in the description of 60 steps in the tool.

\section{ONLINE TOOL}

Apart from:

- the home page (abstract and graphical overview of the design space),

- the bibliography, listing all references that have been taken into account,

- the contact page, for practitioners to send new references,

the tool also provides four main services.

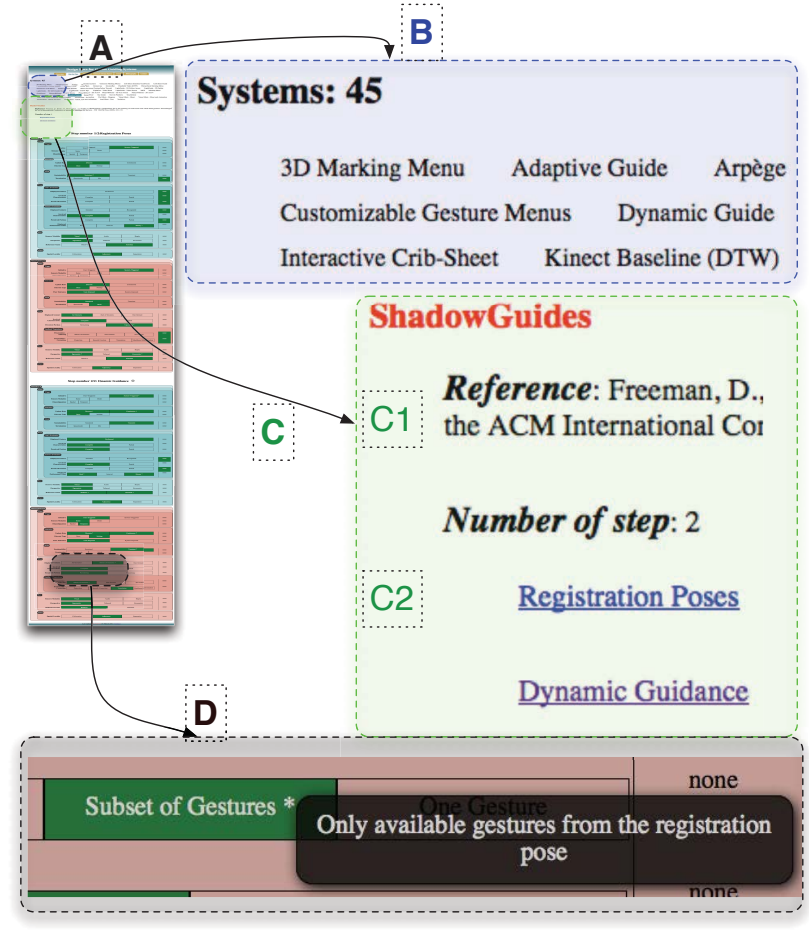

Figure 1: Description of all systems one-by-one (A). First, the practitioner chooses a system among the list of described systems (B). The tool displays the reference of the system (C1). The tool provides the links to the description of the steps (C2). The tool displays the chosen system's step as a green path in the design space (A and D). Some cells includes an asterisk to indicate additional details if hovered over (tooltip in black).

\section{Describing Systems One-By-One}

The tool allows describing of one system at a time (http://iihm.imag.fr/delamare/guidance/desc.html). The tool displays the list of all systems referenced in the tool (Figure $1, \mathrm{~B})$. Once a practitioner clicks on the name of a system, (s)he can see:

1. the reference of the chosen system for further information (Figure 1, C1),

2. the number of steps of the chosen guiding system (Figure 1, C2).

3. a list of links in order to access the description of each step (Figure 1, C2),

4. the description of each step, one after the other (Figure 1, A). For each step, the description is a green colored path within the set of axes corresponding (a) to the feedback mechanism and (b) to the feedforward mechanism. Cells with an asterisk provide a tooltip with additional information about the design option (Figure 1, D).

Because the description based on the design space only concerns the behavior of the guiding system, we think that it is important for the practitioners to have a direct access to the reference in order to obtain complementary information about the visual representation of the guiding system.

The description of each step of a system is useful if the practitioner already knows the guiding system and wants to know the design options while avoiding to read the entire reference again. Thus, this service implements the descriptive power of the design space.

\section{Comparing Systems}

The tool provides a web page for comparing systems (http://iihm.imag.fr/delamare/guidance/compare.html)

(Figure 2, A). This page allows the practitioners to select two or more guiding systems they want to compare (check boxes of Figure 2, B), among 2D, 3D or all guiding systems (radio buttons of Figure 2, B). A table (Figure 2, C) presents the similarity between each step of the compared systems according to the design axes by (a) displaying three similarity scores and by (b) coloring the cell for an overview of the comparison at first glance.

a) Three similarity scores are provided: one for the feedback mechanism, one for the feedforward mechanism and a global one for both mechanisms. These percentages represent the proportion of design options that the compared systems share.

b) Two color scales are provided in order to visually represent the similarity between systems.

The table can be large as it displays all selected systems on both columns and rows to compare them with each other. For helping the exploration of this large table, every cell displays which steps and which systems are compared, in a tooltip when the cursor hovers over it (Figure 2, C).

In order to obtain more detail about similarity scores, the practitioner can click on a cell to visually compare design options within the design space (Figure 3, A) instead of the 


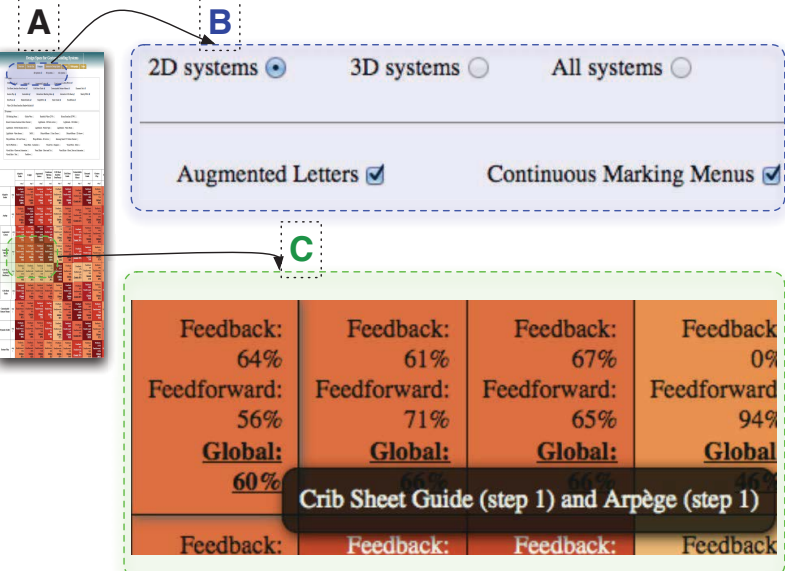

Figure 2: Comparison between systems (A). Selecting the systems to be compared (B). Comparative table with colored cells displaying three similarity scores (feedback mechanism, feedforward mechanism and global scores) (C). On hover, a tooltip recalls the systems and the steps that are compared (C).

comparative table. At the top of this comparison page, the practitioner can see a summary of the two systems (or steps of the systems) that are compared and their similarity scores (Figure 3, B). This summary shows the color code for each system. This color code is used in the design space displayed below the summary (Figure 3, C). In this design space, the design options of the compared systems are represented through colors. If both systems share the same design option, the color of the corresponding cell is a gradient of the two colors representing the two systems.

These two representations for comparison (table or colored paths in the design space) help the practitioner to quickly visualize how similar the systems are. First the overview provided by the colors allows identifying of the systems that are similar and the ones that are unique. Second, closer examination of the representation allows the practitioner to capture the key differences between two guiding systems.

The comparison of two systems with this tool enables the practitioners to fully exploit the descriptive and evaluative powers of the design space. Indeed the tool provides a highlevel visual overview of the comparison. The tool also allows the practitioners to understand the reasons behind differences in performances, if the performances of each compared system are known.

Scenario: Jane wants to experimentally compare two guiding systems. She uses the tool to obtain an overview of the differences between the two guides. She sees that the guides differ according to two axes. Jane then modifies one of the guiding systems so that the two systems differ according to only one axis. After a controlled experiment, she can state which design option is better.

\section{Finding unexplored areas of the design space}

The tool also shows how many systems share a same design option, and this for all design axes of the design space

\section{A}

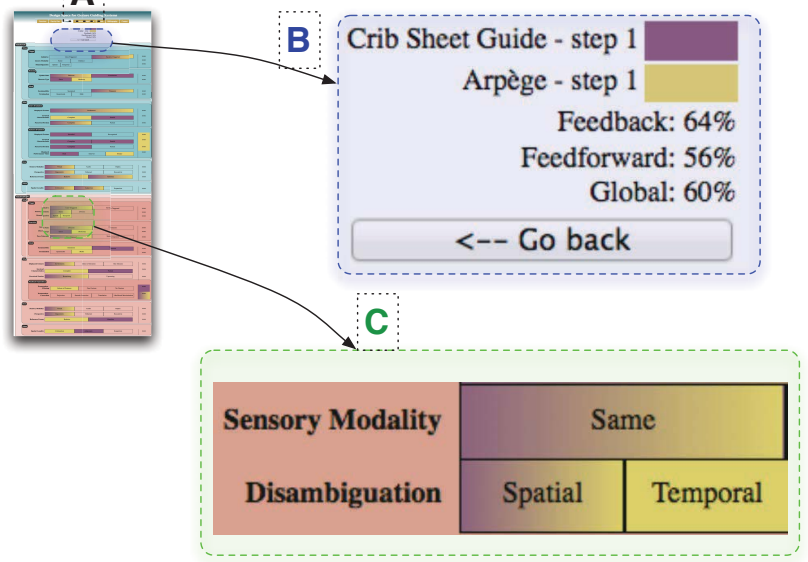

Figure 3: Comparison of two systems represented within the design space (A). Summary of the comparison (B). Colored paths of the two systems (C). Options shared by both systems are filled with a gradient of the two colors representing the two systems (C).

(Figure 4, A). Three cues enable the practitioner to easily benefit from the generative power of the design space: (1) The color of a design option conveys the number of systems (or steps of systems) that implement the design option: the more a design option is implemented by systems, the darker the color of the cell corresponding to the design option is. (http://iihm.imag.fr/delamare/guidance/interactive-designspace.htm and Figure 4). (2) The number of systems implementing the design option is displayed. (3) When the mouse hovers over a cell, a tooltip is displayed and lists all the systems/steps that implement this design option.

By clicking on a design option, practitioners can restrict the presentation to the systems that implement this design option. In the example of Figure 4, the initial state has one filter: the 'Audio' option of the modality in the group How of the feedback mechanism (Figure 4, B). Only four pairs $<$ system/step $>$ implement this design option (Figure 4, C). The design options are highlighted with a green halo (Figure 4, D, the 'Audio' option) so that the practitioner can remember the selected design options. Practitioners can remove this filter by clicking on the cell again.

Scenario 1: Bill is looking for (i) a 3D gesture interaction system. Focusing on the feedforward behavior only, Bill wants to (ii) use the visual modality, show (iii) all gestures (iv) only once. With only four click actions, Bill can see that a system complies with these criteria: 3D Marking Menu [7].

Scenario 2: Jane wants to study (i) 3D gesture guiding systems, which (ii) continuously display the (iii) remaining portion of (iv) all gestures. She notices that no current system actually implements these options. Hence, with only four click actions, she finds a case that has not yet been proposed in the literature. Jane is interested to know if a 2D gesture guiding system with these options exists. She removes the ' $3 \mathrm{D}$ gestures' filter and discovers that a $2 \mathrm{D}$ 


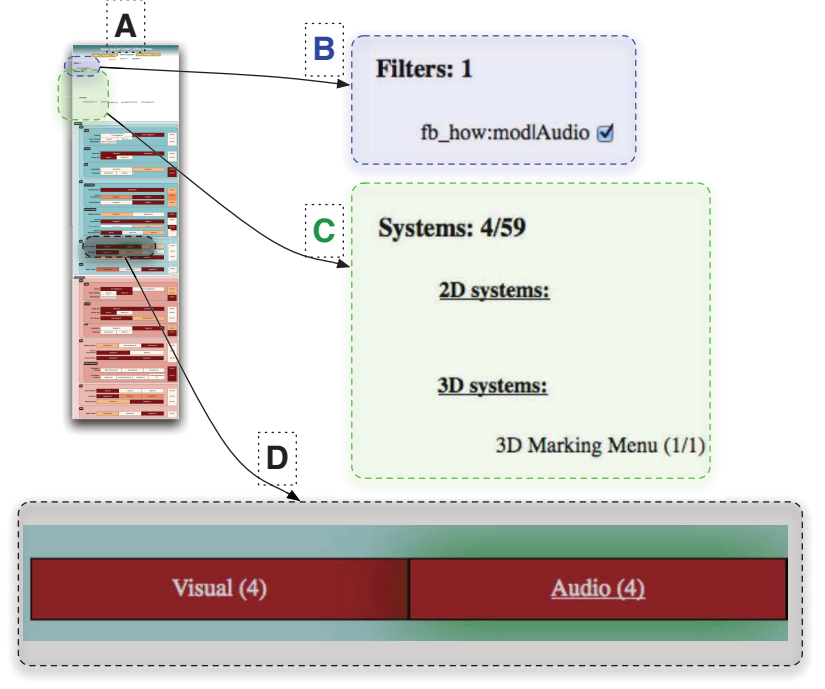

Figure 4: Interactive design space web page (A). In this example, the current state contains one selected filter (B). The tool lists all pairs $<$ system/step $>$ implementing the corresponding design option (C). The practitioner can then visualize in the design space how often the design options are implemented by the other guiding systems (D).

gesture guiding system fulfills her requirements [1]. She has thus identified interesting challenges by applying this solution to $3 \mathrm{D}$ gestures.

This interactive representation helps find out which options are largely implemented, or, on the contrary, underexplored by existing guiding systems. Filtering by a particular design option allows investigating of how design options are combined together. This can end up with (i) a system fulfilling all requirements or (ii), a new system to be developed. Thus, this service assists in benefiting from the generative power of the design space.

\section{Designing a New Guiding System}

The tool provides a web page for designing a guiding system (http://iihm.imag.fr/delamare/guidance/create.html). More specifically, it allows the practitioners to:

1- Enter the number of steps they envision for the guiding system. After validation, the tool will present the corresponding number of representations of the design space - one for each step to design.

2- Click on the cell(s) corresponding to the desired design option(s). Once a design option is set, the cell turns green. The practitioner can remove an option by clicking again on the corresponding cell.

3- Click on the 'Validate' button and compare the resulting guiding system to existing ones. This service is the same as the comparison between systems described above.

This service is valuable in two scenarios. First, it can be used to find an existing guiding system that already meets the specified requirements (i.e. global similarity score of $100 \%$ between the designed new guiding system and an existing one) or to adapt an existing system with the highest similarity score with the new designed system. Second, researchers can use the tool to find guiding systems similar to the new designed system, in order to experimentally compare them and assess the impact of the design options. Thus, this service will promote the evaluative power of the design space in future research.

\section{CONCLUSION}

We have presented an interactive tool for leveraging the use of a design space for gesture guiding systems. The design space, on which the tool is based, aims at characterizing the behavior of gesture guiding systems [5]. It defines 35 axes, 115 design options and currently classifies 46 systems. The number of design options and related systems make it difficult for practitioners to efficiently use this design space. We hence propose this online tool that offers four services. These services enable the practitioners to fully exploit the descriptive, evaluative and generative powers of the design space. It also enhances the capitalization of the knowledge related to the field (i.e. the state-of-the-art and the classification of existing guiding systems) for the practitioners and the researchers.

This demonstration will encourage discussions on improvements of the online tool by its practical use in the context of scenarios and may also enable us to identify new guiding systems to be added in the tool.

\section{ACKNOWLEDGMENTS}

This work has been supported by the DELight project (French government's FUI -Single Inter-Ministry Fundprogram). This work has also been partially supported by the LabEx PERSYVAL-Lab (ANR-11-LABX-0025-01).

\section{REFERENCES}

1. Bau, O. and Mackay, W.E. OctoPocus: A Dynamic Guide for Learning Gesture-Based Command Sets. Proc. UIST '08, ACM Press (2008), 37-46.

2. Beaudouin-Lafon, M.Designing interaction, not interfaces. Proc. AVI '04, ACM Press (2004), 15-22.

3. Card, S.K., Mackinlay, J.D., and Robertson, G.G. A morphological analysis of the design space of input devices. ACM Transactions on Information Systems 9, 2 (1991), 99-122.

4. Cockburn, a., Quinn, P., Gutwin, C., Ramos, G., and Looser, J. Air pointing: Design and evaluation of spatial target acquisition with and without visual feedback. International Journal of Human-Computer Studies 69, 6 (2011), 401-414.

5. Delamare, W., Coutrix, C., and Nigay, L. Designing Guiding Systems for Gesture-Based Interaction. Proc. EICS '15, ACM Press (2015), to appear.

6. Kurtenbach, G., Moran, T.P., and Buxton, W. Contextual Animation of Gestural Commands. Computer Graphics Forum 13, 5 (1994), 305-314.

7. Ren, G. and O'Neill, E. 3D Marking menu selection with freehand gestures. Proc. 3DUI '12, (2012), 61-68. 